arms and hands stretched out on each side of him. The movements before described ceased. At the end of a week, he became quite unconscious, passed urine and fæces under him, and died.

NeCRopsy, thirty-six hours after death.-The body was well nourished; rigor mortis persistent; the thumb of the right hand was drawn into the palm. The cranium was symmetrical ; the calvaria thicker than normal. The dura mater was congested, but easily removed; it was not adherent to the cranium or the cerebrum. The subarachnoid fluid was turbid and in excess; the vessels running over the surface of the brain were congested and the pia mater injected; there was no thickening or opacity of the membranes. A little fluid drained away in removing the brain from the cranial cavity. The brain weighed three pounds and half an ounce; it was firm in consistence. The parietal lobes were thought to be more resistant to pressure than the others. The convolutions were normal in size, with the exception of those in the parietal and temporosphenoidal regions. In the former situation they were slightly, in the latter very coarse in character, i.e., not highly developed. The occipital convolutions were very firm and hard, those on the right side being a little harder than on the left. On slicing through the brain, it was found to be firm ; but the white matter was, if one may so say, "greyish" in appearance. There was a little excess of fluid in the ventricles. The corpus striatum and optic thalamus appeared normal. The white matter of the first temporo-sphenoidal convolution, and of the inferior parietal lobule on both sides, on a level (vertically) with the middle part of the lateral ventricles, appeared fibrous. The same appearance was noticeable in the white matter of the occipital convolutions at the same level..

\section{NOTES ON THE CROTON-OIL TREATMENT OF RINGWORM.}

By ALDER SMITH, M.B.Lond., F.R.C.S., Resident Medical Officer, Christ's Hospital, London.

I SEE in the JOURNAL of May 29th, that the artificial production of kerion is advised as a cure for chronic ringworm. As I have been using croton-oil (in imitation of Nature's cure) for the last six years, and have already fully described my method of producing kerion in my paper on the Diagnosis and Treatment of Ringworm, published in the Lancet of January Ioth, 24th, and 3Ist, I. think it right to advise the profession to be very careful in selecting proper cases for this treatment. In that paper, I strongly recommended the production of an artificial kerion by croton-oil; i.e., "that swollen, raised, inflamed, and infiltrated state of the scalp which sometimes accidentally occurs during treatment, and which always results in a speedy cure of the disease. Kerion should be produced, if possible, in old chronic small patches of ringworm that have resisted all other treatment for many months, but not in those cases where the disease extends over a large extent of surface. The great aim of this treatment is to cause inflammatory swelling and effusion into the tissues around the follicles, so that the stumps, which otherwise would break off on attempted epilation, will now come out with the discharge, or can easily be extracted; in fact, very often in a short time an inveterate patch of ringworm, that has withstood every other treatment for years, can be transformed into a smooth slightly raised place, utterly destitute of all hair and stumps, and practically well." "Even if the swollen condition of kerion cannot be produced, this treatment very rarely fails in loosening the stumps and curing the disease."

I republish these observations of mine, as I should be sorry for the profession to think I advocated the production of kerion indiscriminately in chronic ringworm, especially where a large extent of surface is involved. In fact, the chief cases for which I urge it are those I so constantly see, where, after ordinary treatment for a time, the hair has grown again on the patches, and then the child has been neglected for months or even years, until some special reason brings it under treatment again. "Here the difficulty is not to find some parasiticide that will destroy the ringworm-fungus, but to bring the remedy into contact with it. In recent ringworm, the conidia only penetrate a certain distance into the follicles, and therefore the disease is easily cured by almost any remedy; but after a time they penetrate to the very bottom of the follicles, which, it must be remembered, are even below the true skin." In such cases, it is impossible to reach the fungus by any of the usual remedies applied to the surface of the scalp. Ordinary chronic forms can generally be cured without producing kerion. Painting the places with croton-oil liniment is a good plan; but other remedies will often cause a moderate amount of inflammation, and even slight suppuration, and thus cure the disease. Citrine ointment with carbolic acid (as advised in my paper) will frequently produce this result, especially in yo ng children.
The following, in my experience, are the most suitable cases in which the production of kerion may be attempted.

I. Inveterate cases that have resisted all other treatment for months or years, if not very extensive; especially those where the inveterate parts of the patches have been marked out and reduced in size by other treatment; as by oleate of mercury.

2. Any small patch of ringworm, not larger, say, than half-a-crown, where time is of the utmost importance, and a cure is desired as quickly as possible.

3. Such a case as where ringworm has been detected and properly treated for a time, until the new hair has made its appearance; after which, it has been discontinued, although many diseased stumps remained. Months, or even years, have passed, and the child is perhaps rejected at some public examination. One or more patches are to be seen where the hair is growing freely and firmly, but, on close inspection with a lens, some scurfiness and broken hairs or stumps are observed, scattered among the long hairs on the patches.

Pustulation in minute spots should also be attempted, as probably the only cure for that variety of the disease I described as disseminated ringworm; "one rarely diagnosed, and the most chronic and difficult to cure. The hair is growing freely and firmly all over the head; there are no patches to be seen, although probably they have existed at an earlier stage of the disease; the skin appears generally healthy, and perhaps almost free from scurf, but numerous isolated stumps, or groups of stumps, are seen in every direction, often scattered all over the scalp. This variety is almost always overlooked, and can only be detected by very careful examination. The stumps in these old chronic cases are very brittle, and almost always break on attempted epilation, showing, after soaking some little time in liquor potassæ, under the microscope a most extensive implication with fungus, even down to the root of the hair."

I have often succeeded in curing cases like this when they have resisted all other treatment for years, but they require great care and patience. The entire scalp must be subjected to a very close inspection with a lens, and an attempt made to pull out each stump as it comes into view, and then if it break off, which is most probable, a drop of oil should be applied at once to the follicle, with a very fine sable brush. At the next examination, the oil must again be put on any stumps that break off, as well as upon any fresh ones that appear.

A deep pustular rash alone will often cure chronic ringworm, but certainly not all inveterate cases. My knowledge of such is great, and I know full well, from painful experience, that croton-oil may be painted on time after time, and a pustular rash repeatedly set up, and yet diseased stumps will reappear.

I must remark that it is useless for medical men to attempt to cure very chronic ringworm, especially the disseminated variety, unless they thoroughly realise how intractable some forms of the disease are, possess a good lens and good eyesight, and have plenty of time and patience.

Ringworm must never be considered cured, although the hair has grown again on the patches, as long as a single stump remains affected with the fungus, or any black dots are seen. These black dots are the orifices of diseased follicles, in which the stumps have been broken of on a level with the surface of the scalp by friction, or are the apertures, filled with dirt, left by the retraction of the broken and shortened stump into the follicle after attempted epilation.

It is most difficult to certify that any case of ringworm is absolutely well. Time after time, stumps that were not visible at one examination will crop up again, breaking off when any attempt is made to extract them, and reappearing again and again for months after the case in other respects seems cured. Nor must it be forgotten that stumps are not removed when they only break off, and that no reliance for diagnosis or prognosis can be placed on the microscopical examination of short ordinary hairs taken from a patch, but only of the stumps.

In conclusion, I would warn medical men not to apply croton-oil without due consideration for ordinary cases of chronic ringworm. If they do, they will be sure to get into trouble sooner or later. The oil often causes much inflammation, and parents get frightened, and imagirte the doctor has made the disease ten times worse. Sometimes they will seek other advice, and are told that their former medical attendant has been overtreating the case, and has been using too strong remedies. Under soothing applications, the little patient gets well, and the second attendant gets the credit of curing the ringworm, which was practically well when he first saw the case. I always explain to parents beforehand the reasons for adopting this treatment, and the results to be expected from it. It is also important to bear in mind that simple remedies will generally be efficacious in eradicating ringworm in young children, and that stronger ones in such cases should never be employed. 